\title{
ANEURYSMAL BONE CYST OF CALCANEUM WITH PATHOLOGICAL FRACTURE: A CASE REPORT
}

Swagat Mahapatra ${ }^{1}$, Suruchi²

\section{HOW TO CITE THIS ARTICLE:}

Swagat Mahapatra, Suruchi. "Aneurysmal Bone Cyst of Calcaneum with Pathological Fracture: A Case Report". Journal of Evolution of Medical and Dental Sciences 2014; Vol. 3, Issue 24, June 16; Page: 6776-6780, DOI: $10.14260 /$ jemds/2014/2818

\begin{abstract}
Aneurysmal Bone cyst is a benign solitary lesion of unknown etiology. It occurs mostly in long bones, very rarely (3\% of cases) it occurs in the foot bones that too in the calcaneum, it is extremely rare. CASE: A twenty-one years old male with history of a slow growing swelling on right heel since two years and dull aching pain over the swelling. There was history of trivial trauma to same heel for which patient was treated with a boot cast by a local doctor. Investigations were done and X-ray showed central multiloculated lytic lesion with bone destruction and no new bone formation with trabeculations. FNAC revealed blood elements indicating towards Aneurysmal bone cyst. Excision was planned and per-operative finding was welling of blood at operation site. Extensive curettage followed by corticocancellous bone grafting was done and patient was discharged with a boot cast. On follow-up the grafts were taken up well. Weight bearing was started after 10 weeks. Patient was followed up for 2 years. There was no sign of recurrence. The lytic lesion healed well. CONCLUSION: $\mathrm{ABC}$ of calcaneum along with pathological fracture is very rare.

KEYWORDS: Calcaneum - Aneursmal bone cyst -Pathological fracture.
\end{abstract}

INTRODUCTION: $\mathrm{ABC}$ is an expansile cystic lesion that affects individuals during their $2^{\text {nd }}$ decade of life and may occur in any bone in the body. Although benign, ABC can be locally aggressive and can cause extensive weakening of the bony structures and impinge on the surrounding tissues. ABCs both erode and cause expansion of underlying cancellous and cortical bone. Around the lesion there is always a shell formed by periosteal new bone and although it may be only be a millimeter thick, it prevents direct extension into the soft tissues. The expansile nature of this lesion can cause pain, swelling, deformity, disruption of growth plate, neurologic symptoms and least commonly pathological fracture.

$\mathrm{ABC}$ in the foot are very uncommon. ABC's constitute $1 \%$ of all primary bone tumors and occurrence in the foot is only about $3 \%$ compared to other bones ${ }^{1}$. Occurrence within the calcaneum is rare and present as chronic heel pain and swelling but may rarely present as pathologic fracture. A plethora of cystic lesions can occur in the calcaneum, which makes definitive diagnosis difficult based on imaging only. The differential diagnosis includes simple bone cyst, ABC (primary or secondary), chondroblastoma, giant cell tumor (GCT), osteosarcoma, ossifying hematoma or pseudotumor of hemophilia. This mandates histopathological diagnosis prior to the definitive management.

We report a rare case of $\mathrm{ABC}$ involving calcaneum of 21years old male confirmed by histopathology report and we performed curettage and bone grafting of cyst.

CASE REPORT: A twenty-one years old male with history of a slow growing swelling on right heel since two years and dull aching pain over the swelling, gave history of trivial trauma to same heel for which patient was treated with a boot cast by a local doctor. Clinical evaluation revealed swelling 
over the lateral aspect of the heel and the skin over the swelling was stretched. Tenderness was present on palpation but there was no local rise of temperature. The swelling was bony hard in consistency and arising from calcaneum. There were no distal neurovascular deficits or any significant lymphadenopathy.

Investigations were done and radiographic examination of his ankle revealed an eccentric, expansile, multiloculated lytic lesion of the calcaneum with thin trabeculae traversing the cystic cavity. There was no breach in the cortex. Based on clinical and radiological findings, a diagnosis of benign cystic lesion of right calcaneum was made.

Open biopsy of the cyst was made to confirm the diagnosis. The cyst grossly consisted of cavities filled with brown altered blood. Histopathological report revealed large blood filled cavities lined by fibrous septa, with occasional osteoclastic giant cells. Hence the diagnosis of ABC involving the calcaneum was made.

The patient was scheduled for cyst curettage and bone grafting. By curvilinear incision over the lateral aspect of heel, the calcaneum was exposed. A large cortical window was made and the entire cyst curettage was done. Then the cavity was washed with saline and packed with corticocancellous bone graft harvested from both iliac crests in addition to synthetic bone substitute. The patient was advised non-weight bearing walking on the affected limb for eight weeks.

Later mobilized with partial weight bearing walking for a further four weeks and then followed by full weight bearing on affected limb. At six months of follow up, the patient was pain free and had returned to his regular activities. At two years follow-up, the patient is clinically asymptomatic. There is no evidence of recurrence.

DISCUSSION: $A B C$ is an entity on its own having unique clinical, radiological and diagnostic behavior. The true etiology of ABCs is unknown ${ }^{2}$. Most investigators believe that $\mathrm{ABC}$ are the result of a vascular malformation within the bone; however, the ultimate cause of the malformation is a topic of controversy. The concept of an $\mathrm{ABC}$ as a secondary phenomenon occurring in a pre-existing lesion is based on the fact that in approximately one-third of the cases a pre -existing lesion can be identified, the most common of which is giant-cell tumor. ABCs are common around the knee joint of the young and have an equal incidence in both genders.

About $50-70 \%$ of ABCs occur in the second decade of life, with 70-86\% occurring in patients younger than 20 years $^{3}$, which makes this case even more unusual. On histology, the $A B C$ is characterized by blood filled cavities lined by fibrous septa. The stroma contains proliferative fibroblasts, spindle cells, areas of osteoid formation, and an uneven distribution of multinucleated giant cells. The tissue within the septations includes cavernous channels that do not contain a muscular or elastic layer in their walls.

Areas of new and reactive bone formation can also be found in the ABC. Mitotic figures are common to ABCs, but no atypical figures should be evident ${ }^{4}$. Bone cysts of the calcaneum are rare lesions. These may include a wide spectrum of non-neoplastic cysts, benign or malignant neoplastic lesions ranging from simple bone cyst, ABC (primary or secondary), chondroblastoma, giant cell tumor (GCT), and an osteosarcoma (especially telangiectatic).

Clinically, calcaneal cysts are often symptomatic and present with heel pain, although some of these lesions may remain asymptomatic and are detected as incidental findings. Even though there are many typical radiograph, computed tomographic (CT) scan and magnetic resonance imaging 
(MRI) findings to confirm a diagnosis of $\mathrm{ABC}$, an open biopsy must be performed because of the high frequency of accompanying tumors ${ }^{5}$.

When a biopsy is performed, the sample should ideally include material from the entire lesion. A limited biopsy could easily cause a coexisting lesion to be missed, leaving the patient with a morbid prognosis. There are various methods of treatment based on the site and size of the lesion, which include curettage, which may be supplemented with various adjuvant therapies such as bone grafting, use of liquid nitrogen, phenol instillation and Poly (methyl methacrylate) (PMMA) cement ${ }^{6}$.

Despite a favorable outcome of ABCs with an overall cure rate of $90-95 \%$, one of the most common problems encountered during management is frequent recurrence. The incidence of recurrence has been noted to vary between $59 \%$ in cases treated with intralesional excision and $0 \%$ in cases with resection. Recurrence usually happens within the first year after surgery, and almost all episodes occur within 2 years. Therefore, a patient of $A B C$ needs to be observed for at least this period of time to exclude any recurrence. It is beneficial to detect recurrence early when the lesion is still small and easier to treat.

To conclude, $\mathrm{ABC}$ of the calcaneum is an extremely uncommon entity ${ }^{7}$. Proper diagnosis entails correlating the clinical presentation, anatomical location, radiological profile, and histopathological appearance. This is imperative not only to exclude other more common histological mimics, but also for choosing the appropriate therapeutic regimen a prognosticating the disease outcome. In a case of calcaneal cystic lesion, ABC should be considered as one of the differential diagnosis. Hence histological diagnosis is essential. Curettage and bone grafting is a valuable option ${ }^{8}$.

\section{REFERENCES:}

1. Clayer M. Injectable form of calcium sulphate as treatment of aneurysmal bone cysts. ANZ J Surg 2008 78(5): 366-370.

2. Segall L, Cohen-Kerem R, Ngan B Y, Forte V. Aneurysmal bone cysts of the head and neck in pediatric patients: A caseseries. Int J Pediatr Otorhinolaryngol 21 2008: epub ahead of print.

3. Burch S, Hu S, Berven S. Aneurysmal bone cysts of the spine. Neurosurg Clin N Am 200819 (1): 41-47.

4. Brastianos P, Gokaslan Z, Mc Carthy E F. Aneurysmal bone cysts of the sacrum: a report of ten cases and review of the literature. Iowa Orthop J 2009 29: 74-78.

5. Sun Z J, Zhao Y F, Yang R L, Zwahlen R A. Aneurysmal Bone Cysts of the Jaws: Analysis of 17 Cases. J Oral Maxillofac Surg Jan 262010 (Medline).

6. Jaffe H L, Lichtenstein L. Solitary unicameral bone cyst with emphasis on the roentgen picture, the pathologic appearance and the pathogenesis. Arch Surg 1942 44: 1004-1025.

7. Campanacci M, Capanna R, Picci P. Unicameral and aneurysmal bone cysts. ClinOrthop 1986 204: 25-36.

8. Enneking WF. Aneurysmal bone cyst. In: Musculoskeletal tumor surgery. New York: Churchill Livingstone, 1983; 1513-29. 


\section{CASE REPORT}
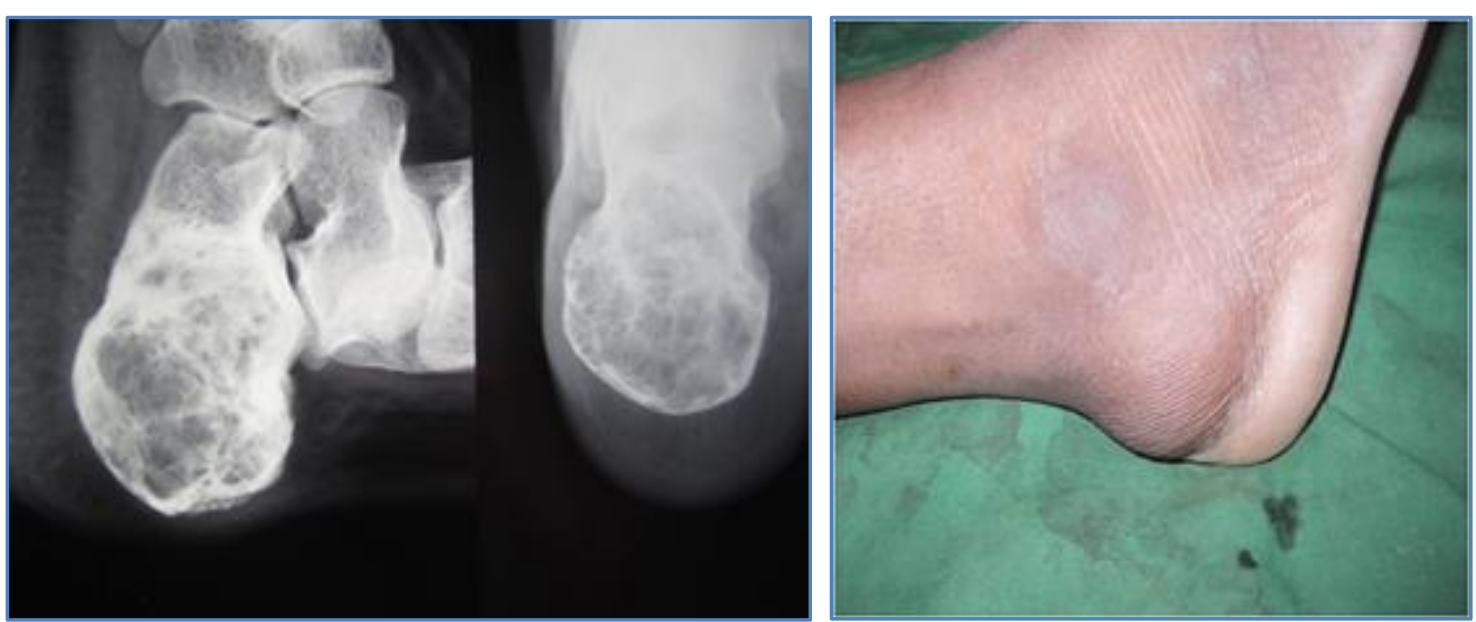

Pre - operative
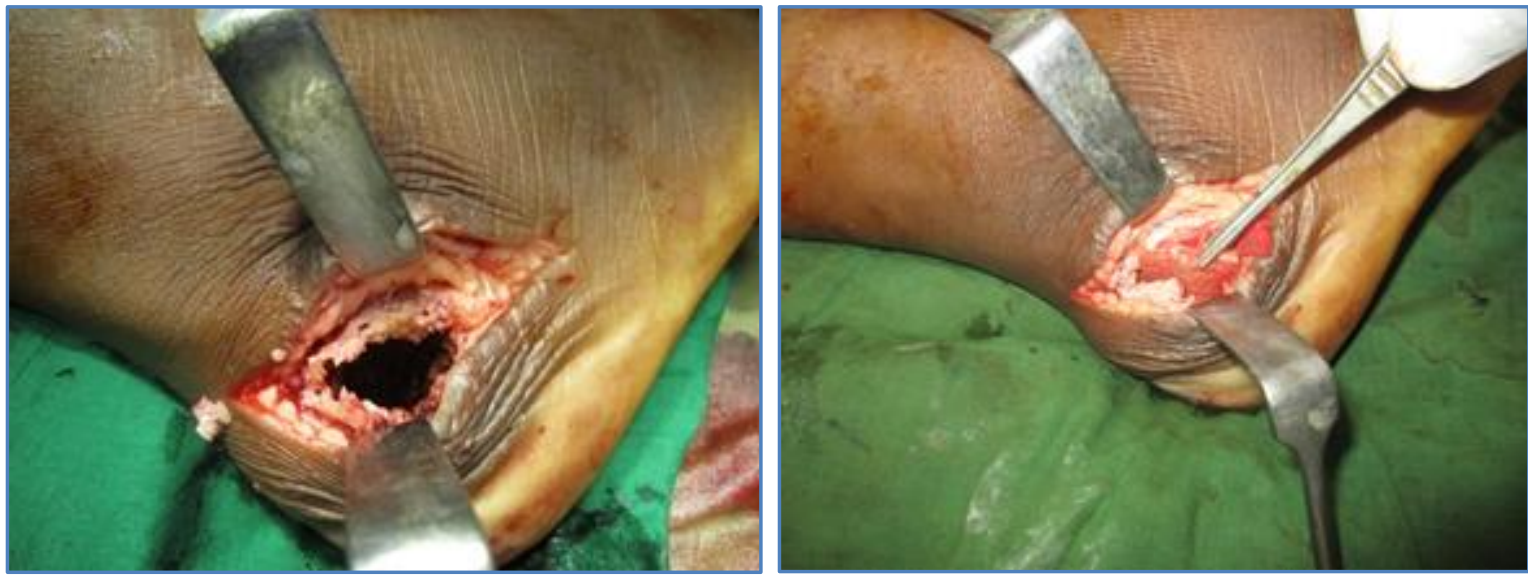

INTRA - OP
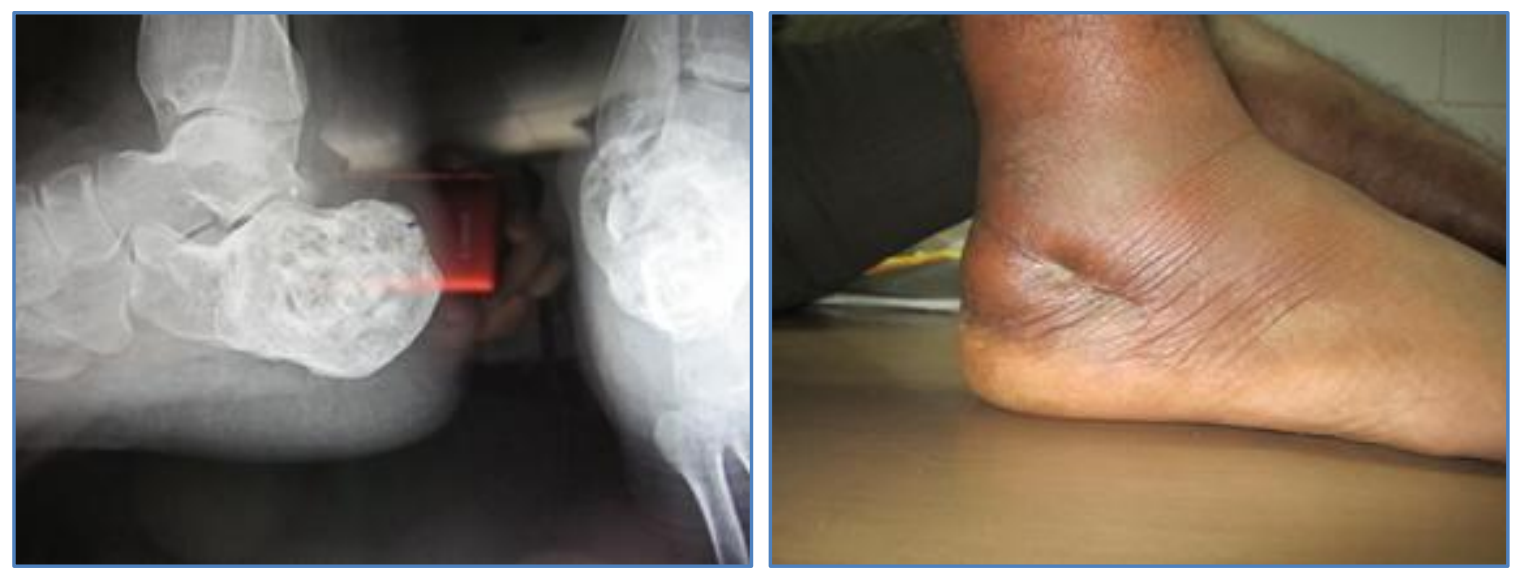

Post- operative- 1 Year 


\section{CASE REPORT}

\section{AUTHORS:}

1. Swagat Mahapatra

2. Suruchi

\section{PARTICULARS OF CONTRIBUTORS:}

1. Senior Resident, Department of Orthopaedics, JIPMER.

2. Senior Resident, Department of Anaesthesia, JIPMER.

\section{NAME ADDRESS EMAIL ID OF THE} CORRESPONDING AUTHOR:

Dr. Swagat Mahapatra, Old MSR Quarters,

No. 21, JIPMER Campus,

Pondicherry-605006,

Email: drswagat@gmail.com

Date of Submission: 19/05/2014.

Date of Peer Review: 20/05/2014.

Date of Acceptance: 07/06/2014.

Date of Publishing: 16/06/2014. 